\title{
Could boosting the oligoadenylate synthetase-like pathway bring a new era of antiviral therapy?
}

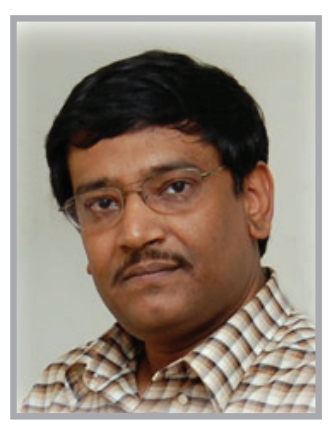

\section{Saumendra N Sarkar*}

Viruses present a major threat to global health with significant pandemic potential. Among different types of viruses that are human pathogens, RNA viruses pose unique challenges due to their rapid replication kinetics, high mutation rates and complex evolutionary dynamics. The coevolution of the virus and the host has resulted in competing strategies to protect and propagate over a long time. As a result, oftentimes, the host antiviral systems that prevent virus infection are targeted by viruses [1]. Therefore, understanding the inner workings of the host innate immune system - the first line of defense against viral infection, and how it is subverted by viruses gives us one of the most promising opportunities to combat diseases caused by RNA viruses. In this context, recent elucidation of the mechanism of antiviral activity of one such host proteins oligoadenylate synthetase-like (OASL) has shown the potential for developing broad acting antiviral therapy exploiting this pathway [2].

\section{Viral RNA sensing \& IFN induction}

Cellular innate immunity, triggered by sensing of viral RNA through specialized receptors, regulates not only the outcome of a viral infection, but also tissue tropism, predisposition to disease, inflammation and tumorigenesis [3]. Following entry into target cells, viral RNA is detected by cytosolic receptors such as RIG-I-like receptors (RLR) and Toll-like receptors (TLR) through the detection of nonself nucleic acids. RLR engagement with viral RNA leads to activation of signaling pathways resulting in transcriptional induction of type I interferons (IFNs). IFN induced by RLR or TLR signaling acts in an autocrine or paracrine manner to induce many genes, together called IFN-stimulated genes (ISG). Due to the common transcriptional elements in their promoters, several ISGs are also targets for direct induction by viral infection (via IRF3/ IRF7), without requiring IFN signaling. Most of the pleotropic effects of IFN are mediated by ISGs. The best-understood mechanism for the antiviral activity of ISG is the generalized inhibition of protein synthesis by dsRNA-activated enzymes (e.g., RNA-activated protein kinase [PKR], oligoadenylate synthetase [OAS], and so on). However, among approximately 400 ISGs, the biochemical function of only a handful has been delineated [4]. Recently, a number

\section{KEYWORDS}

- influenza • interferon • OASL

- RIG-I

*Cancer Virology Program, University of Pittsburgh Cancer Institute \& Department of Microbiology \& Molecular 
"It has been shown that the synthesis of short K63-linked polyubiquitin chains, or the K63-linked polyubiquitination of RIG-I,

is carried out by the ubiquitin ligase TRIM25.” of ISGs were tested for their antiviral activities against a variety of viruses [5]. However, the mechanisms of antiviral activity for most of these genes remain unknown.

\section{IFN-inducible oligoadenylate synthetase- like protein}

OAS is a family of ISGs characterized by their ability to synthesize $2^{\prime}-5^{\prime}$ oligoadenylates, which induce RNA degradation by activating a latent RNase, RNaseL [6]. However, the recent identification of the cytoplasmic DNA sensor cyclic GMP-AMP synthetase (cGAS), which is another member of OAS family, shows potentially diverse functions of this family of proteins [7]. Human OAS-like (OASL) is related to the OAS family by its $\mathrm{N}$-terminal OAS-like domain, which seems to be derived from an ancestral OAS protein, but harbors characteristic changes in the active site, and is thus devoid of 2'-5' OAS activity. Furthermore, OASL contains two tandem ubiquitin-like domains (UBL) in the C-terminus that are not present in any of the other members of the OAS family [6]. $O A S L$ is directly and rapidly induced by virus infection via IFN regulatory factor (IRF) -3 as well as by IFN signaling and has been shown to have antiviral activities, which requires the UBL domain [8]. We have recently demonstrated that OASL interacts with RIG-I and enhances RIG-I-mediated IFN induction [2].

Unlike in humans, two $O A S L$ orthologs have been identified in mice: Oasll and Oasl2, sharing respectively 70 and $48 \%$ amino acid sequence identity with human $O A S L$ [6]. Uniquely, the mouse Oasl 2 contains two crucial Asp residues in its active site and it exhibits OAS enzyme activity. The mouse Oasl1, has been recently shown to inhibit IFN induction by binding to the $5^{\prime}$ untranslated region (UTR) of IRF7 and inhibiting its translation. Consequently, targeted deletion of Oasl1 led to enhanced IFN induction and diminished viral replication in vivo [9]. In contrast to Oasl1, human OASL and mouse Oasl2 do not bind to the IRF7 5'UTR and are devoid of IRF7 suppression activity. Targeted deletion of Oasl2 in mice showed enhanced virus replication suggesting that Oasl 2 acts as the functional equivalent of human OASL.

\section{Mechanism of OASL action}

OASL promotes antiviral activity by enhancing the sensitivity of RIG-I activation. From a number of biochemical and structural studies [10], a model for RIG-I activation has been proposed where RIG-I adopts a stable auto-inhibited conformation in the absence of RNA. Upon binding to viral RNA through the C-terminal domain, the helicase domain changes conformation, thereby enabling OASL to hydrolyze ATP and further interact with RNA. The N-terminal caspase activation and recruitment domains (CARDs) then bind to K63-linked polyubiquitin (pUb), converting RIG-I to an active competent state, which is followed by CARD-mediated MAVS aggregation and signaling. Recent observations also suggest RNA-dependent RIG-I aggregation in the case of longer RNA resulting in promotion of MAVS activation [11]. Although for larger dsRNA the strict requirement of $\mathrm{pUb}$ for RIG-I activation has been a topic of debate, in most cases RIG-I activation is strongly regulated by a two-step mechanism requiring simultaneous binding of two ligands - RNA and pUb. This mechanism makes the RIG-I sensor likely to avoid aberrant activation of antiviral innate immunity and IFN induction. It has been shown that the synthesis of short K63-linked polyubiquitin chains, or the K63-linked polyubiquitination of RIG-I, is carried out by the ubiquitin ligase TRIM25 [12,13]. However, we have shown that in presence of OASL RIG-I can be activated by viral RNA in the absence of TRIM25 [2]. Thus, we believe that following the initial viral infection and OASL induction in the infected and the surrounding cells through IFN signaling, OASL binds to RIG-I and mimics pUb. This makes RIG-I activation more sensitive requiring just one ligand, viral RNA and leads to enhanced IFN induction.

\section{Subversion of innate host defense by viruses}

As the primary mediators of antiviral innate immunity, the RLR and the IFN pathways are targeted by multiple RNA viruses. Influenza virus, the causative agent for seasonal influenza outbreaks and associated mortalities, accomplishes this by the nonstructural protein, NS1 through various mechanisms [14]. NS1 can directly bind RIG-I and/or limit ligand availability by binding RNA $[15,16]$. Further, NS1 can attenuate activation of RIG-I via inhibition of ubiquitination by the ubiquitin ligase, TRIM25 [12]. As it has been shown that in presence of OASL RIG-I activation can be carried out without TRIM25, it is expected that boosting OASL expression may provide strong 
antiviral activity against all strains of influenza viruses. Similarly, another respiratory pathogen respiratory syncytial virus targets various components of RLR and IFN pathway [17]. However, in presence of OASL, this subversion is substantially attenuated [SARKAR SN ET AL., UnPUBLISHED DATA] providing a strong antiviral activity [2]. Among the positive strand RNA viruses, HCV, which is sensed through RIG$\mathrm{I}$, is again inhibited by OASL [8]. However, the picorna viruses that are primarily sensed through MDA5 (another member of the RLR family) are not inhibited by OASL showing specificity. Interestingly, OASL is not targeted by picorna viruses to subvert innate immunity. These findings together argue in favor of using OASL to provide broad antiviral activity against viruses that are primarily sensed through RIG-I, which might help overcome viral subversion of innate immunity.

\section{Unmet needs \& how OASL may be useful}

Two aspects about OASL-mediated enhancement of RIG-I signaling make it unique for combating virus infection. First, OASL has the potential to overcome the innate immune evasion. According to our results with influenza virus, despite targeting of TRIM25 by NS1, RIG-I can be activated in presence of OASL [Sarkar SN ET AL., Unpublished data]. Second, unlike RIG-I expression, which results in IFN induction that can lead to toxicity, expression of OASL by itself does not activate IFN induction. It makes the RIG-I-based RNA detection system much more sensitive to viral RNA, where it can be activated with comparatively subthreshold levels of virus infection [2].
Therefore, delivering OASL protein or ectopically expressing OASL is less likely to have major toxic side effects and may prove a new mode of combating virus infection. However, successful delivery of a cytoplasmic protein to obtain therapeutic efficacy is a daunting challenge for the current drug delivery technologies. Thus, we are focusing on the respiratory viruses, which affect the respiratory system that is much more accessible to noninvasive manipulations. In case of influenza the primary focus of protection against influenza on a global scale is centered upon vaccine development. Vaccination efforts have resulted in a reduction of seasonal illness related to influenza, but are limited in efficacy overall. The emergence of novel strains presents limits on vaccine generation, resulting in a large rate of infection prior to the availability of vaccine. OASL presents a novel molecule that may be able to boost innate host defense, even in the presence of viral inhibition, resulting in improved immunity. Successful development of OASL-based therapy against influenza may further allow us to apply the same principle and technologies for combating other RNA viruses.

\section{Financial \& competing interests disclosure}

Research in the author's laboratory is supported by NIH funding grant AI082673 and in part by award P30CA047904 to University of Pittsburgh Cancer Institute. The author has no other relevant affliations or financial involvement with any organization or entity with a financial interest in or financial conflict with the subject matter or materials discussed in the manuscript apart from those disclosed.

No writing assistance was utilized in the production of this manuscript.

\author{
“The emergence of novel \\ strains presents limits on \\ vaccine generation, \\ resulting in a large rate of \\ infection prior to the \\ availability of vaccine."
}

\section{References}

1 Bowie AG, Unterholzner L. Viral evasion and subversion of pattern-recognition receptor signalling. Nat. Rev. Immunol. 8(12), 911-922 (2008).

2 Zhu J, Zhang Y, Ghosh A et al. Antiviral activity of human OASL protein is mediated by enhancing signaling of the RIG-I RNA sensor. Immunity 40(6), 936-948 (2014).

3 Rouse BT, Sehrawat S. Immunity and immunopathology to viruses: what decides the outcome? Nat. Rev. Immunol. 10(7), 514-526 (2010).

4 Sadler AJ, Williams BR. Interferon-inducible antiviral effectors. Nat. Rev. Immunol. 8(7), 559-568 (2008).
5 Schoggins JW, Macduff DA, Imanaka N et al. Pan-viral specificity of IFN-induced genes reveals new roles for cGAS in innate immunity. Nature 505(7485), 691-695 (2014).

6 Kristiansen H, Gad HH, Eskildsen-Larsen S, Despres P, Hartmann R. The oligoadenylate synthetase family: an ancient protein family with multiple antiviral activities. J. Interferon Cytokine Res. 31(1), 41-47 (2011).

7 Sun L, Wu J, Du F, Chen X, Chen ZJ. Cyclic GMP-AMP synthase is a cytosolic DNA sensor that activates the type I interferon pathway. Science 339(6121), 786-791 (2013).

8 Schoggins JW, Wilson SJ, Panis M et al. A diverse range of gene products are effectors of the type I interferon antiviral response. Nature 472(7344), 481-485 (2011).

9 Lee MS, Kim B, Oh GT, Kim YJ. OASL1 inhibits translation of the type I interferonregulating transcription factor IRF7. Nat. Immunol. 14(4), 346-355 (2013).

10 O'Neill LA, Bowie AG. The powerstroke and camshaft of the RIG-I antiviral RNA detection machine. Cell 147(2), 259-261 (2011).

11 Peisley A, Wu B, Yao H, Walz T, Hur S. RIG-I forms signaling-competent filaments in an ATP-dependent, ubiquitin-independent manner. Mol. Cell 51(5), 573-583 (2013).

12 Gack MU, Albrecht RA, Urano T et al. Influenza A virus NS1 targets the ubiquitin ligase TRIM25 to evade recognition by the 


\section{EDITORIAL Sarkar}

host viral RNA sensor RIG-I. Cell Host Microbe 5(5), 439-449 (2009).

13 Zeng W, Sun L, Jiang X et al. Reconstitution of the RIG-I pathway reveals a signaling role of unanchored polyubiquitin chains in innate immunity. Cell 141(2), 315-330 (2010).

14 Fernandez-Sesma A. The influenza virus NS1 protein: inhibitor of innate and adaptive immunity. Infect. Disord. Drug Targets 7(4), 336-343 (2007).

15 Mibayashi M, Martinez-Sobrido L, Loo YM, Cardenas WB, Gale M Jr, Garcia-Sastre A. Inhibition of retinoic acid-inducible gene I-mediated induction of beta interferon by the NS1 protein of influenza A virus. J. Virol. 81(2), 514-524 (2007)
16 Pichlmair A, Schulz O, Tan CP et al. RIG-I-mediated antiviral responses to single-stranded RNA bearing 5'-phosphates. Science 314(5801), 997-1001 (2006).

17 Barik S. Respiratory syncytial virus mechanisms to interfere with type 1 interferons. Curr. Top Microbiol. Immunol. 372, 173-191 (2013). 